\title{
Flavonoid and lignan intake in relation to bladder cancer risk in the European Prospective Investigation into Cancer and Nutrition (EPIC) study
}

R Zamora-Ros ${ }^{\star}, 1,2$, C Sacerdote ${ }^{3}$, F Ricceri ${ }^{3}$, E Weiderpasss ${ }^{4,5,6,7}$, N Roswall ${ }^{8}$, G Buckland ${ }^{1}$, D E St-Jules ${ }^{9}$, K Overvad $^{10}$, C Kyrø 8 , G Fagherazzi ${ }^{11,12,13}$, M Kvaskoff ${ }^{11,12,13}$, G Severi ${ }^{14,15}$, J Chang-Claude ${ }^{16}$, R Kaaks ${ }^{16}$, U Nöthlings ${ }^{17}$, A Trichopoulou ${ }^{18,19}$, A Naska ${ }^{20}$, D Trichopoulos ${ }^{18,19,21}$, D Palli ${ }^{22}$, S Grioni $^{23}$, A Mattiello ${ }^{24}$, R Tumino ${ }^{25}$, I T Gram ${ }^{4}$, D Engeset ${ }^{4}$, J M Huerta ${ }^{26,27}$, E Molina-Montes ${ }^{27,28}$, M Argüelles ${ }^{29}$, P Amiano ${ }^{27,30,}$

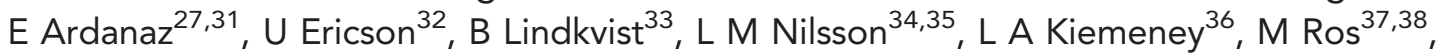
H B Bueno-de-Mesquita ${ }^{39,40,41}$, P H M Peeters ${ }^{41,42}$, K-T Khaw ${ }^{43}$, N J Wareham ${ }^{44}$, V Knaze ${ }^{2}$, I Romieu ${ }^{2}$, A Scalbert ${ }^{2}$, P Brennan ${ }^{45}, \mathrm{P}$ Wark ${ }^{41}, \mathrm{P}$ Vineis ${ }^{41}$, E Riboli ${ }^{41}$ and C A González ${ }^{1}$

${ }^{1}$ Unit of Nutrition, Environment and Cancer, Catalan Institute of Oncology (ICO), Bellvitge Biomedical Research Institute (IDIBELL), Barcelona, Spain; ${ }^{2}$ Section of Nutrition and Metabolism, International Agency for Research on Cancer (IARC), Lyon, France; ${ }^{3}$ Center for Cancer Prevention (CPO-Piemonte), and Human Genetic Foundation (HuGeF), Torino, Italy; ${ }^{4}$ Department of Community Medicine, University of Tromsø, The Arctic University of Norway, Tromsø, Norway; ${ }^{5}$ Cancer Registry of Norway, Oslo, Norway; ${ }^{6}$ Department of Medical Epidemiology and Biostatistics, Karolinska Institutet, Stockholm, Sweden; ${ }^{7}$ Samfundet Folkhälsan, Helsinki, Finland; ${ }^{8}$ Danish Cancer Society Research Center, Copenhagen, Denmark; ${ }^{9}$ Department of Nutrition, Harvard School of Public Health, Boston, MA, USA; ${ }^{10}$ Department of Public Health, Section for Epidemiology, Aarhus University, Aarhus, Denmark; ${ }^{11}$ Inserm, Centre for research in Epidemiology and Population Health (CESP), U1018, Nutrition, Hormones and Women's Health team, Villejuif, France; ${ }^{12}$ Paris South University, UMRS 1018, Villejuif, France; ${ }^{13}$ IGR, F-94805, Villejuif, France; ${ }^{14}$ Cancer Epidemiology Centre, Cancer Council Victoria, Melbourne, Victoria, Australia; ${ }^{15}$ Centre for Molecular, Environmental, Genetic, and Analytic Epidemiology, The University of Melbourne, Melbourne, Victoria, Australia; ${ }^{16}$ Division of Cancer Epidemiology, German Cancer Research Center, Heidelberg, Germany; ${ }^{17}$ Department of Nutrition and Food Sciences, Nutritional Epidemiology, University of Bonn, Bonn, Germany; ${ }^{18}$ Hellenic Health Foundation, Athens, Greece; ${ }^{19}$ Bureau of Epidemiologic Research, Academy of Athens, Athens, Greece; ${ }^{20}$ Department of Hygiene, Epidemiology and Medical Statistics, University of Athens Medical School, Athens, Greece; ${ }^{21}$ Department of Epidemiology, Harvard School of Public Health, Boston, MA, USA; ${ }^{22}$ Molecular and Nutritional Epidemiology Unit, Cancer Research and Prevention Institute-ISPO, Florence, Italy; ${ }^{23}$ Nutritional Epidemiology Unit, Fondazione IRCCS Istituto Nazionale dei Tumori, Milan, Italy; ${ }^{24}$ Dipartimento di Medicina Clinica e Chirurgia, Federico II University, Naples, Italy; ${ }^{25}$ Cancer Registry and Histopathology Unit, 'Civic MP Arezzo' Hospital, ASP Ragusa, Italy; ${ }^{26}$ Department of Epidemiology, Murcia Regional Health Council, Murcia, Spain; ${ }^{27}$ CIBER Epidemiología y Salud Pública (CIBERESP), Madrid, Spain; ${ }^{28}$ Andalusian School of Public Health, Granada, Spain; ${ }^{29}$ Public Health Directorate, Asturias, Spain; ${ }^{30}$ Public Health Department of Gipuzkoa, BioDonostia Research Institute, Health Department of Basque Region, San Sebastián, Spain; ${ }^{31}$ Public Health Institute of Navarra, Pamplona, Spain; ${ }^{32}$ Diabetes and Cardiovascular disease, Genetic Epidemiology, Department of Clinical Sciences, Lund University, Malmö, Sweden; ${ }^{33}$ Institute of Medicine, Sahlgrenska Academy, University of Gothenburg, Gothenburg, Sweden; ${ }^{34}$ Department of Public Health and Clinical Medicine, Umeå University, Umeå, Sweden;

*Correspondence: Dr R Zamora-Ros; E-mail: rzamora@iconcologia.net

Received 3 March 2014; revised 16 July 2014; accepted 20 July 2014; published online 14 August 2014

(c) 2014 Cancer Research UK. All rights reserved 0007-0920/14 
${ }^{35}$ Arcum, Arctic Research Centre at Umeå University, Umeå, Sweden; ${ }^{36}$ Department for Health Evidence and Department of Urology, Radboud University Medical Center, Nijmegen, The Netherlands; ${ }^{37}$ National Institute for Public Health and the Environment (RIVM), Bilthoven, The Netherlands; ${ }^{38}$ Department of Epidemiology, Biostatistics and HTA, Radboud University Nijmegen Medical Centre, Nijmegen, The Netherlands; ${ }^{39}$ National Institute for Public Health and the Environment (RIVM), Bilthoven, The Netherlands; ${ }^{40}$ Department of Gastroenterology and Hepatology, University Medical Centre, Utrecht, The Netherlands; ${ }^{41}$ School of Public Health, Imperial College, London, UK; ${ }^{42}$ Department of Epidemiology, Julius Center for Health Sciences and Primary Care, University Medical Center Utrecht, Utrecht, The Netherlands; ${ }^{43}$ Department of Public Health and Primary Care, University of Cambridge, Cambridge, UK; ${ }^{44}$ MRC Epidemiology Unit, Cambridge University, Institute of Metabolic Science, Cambridge, UK and ${ }^{45}$ Genetic Epidemiology Group, Section of Genetics, International Agency for Research on Cancer (IARC), Lyon, France

Background: There is growing evidence of the protective role of dietary intake of flavonoids and lignans on cancer, but the association with bladder cancer has not been thoroughly investigated in epidemiological studies. We evaluated the association between dietary intakes of total and subclasses of flavonoids and lignans and risk of bladder cancer and its main morphological type, urothelial cell carcinoma (UCC), within the European Prospective Investigation into Cancer and Nutrition (EPIC) study.

Methods: A cohort of 477312 men and women mostly aged 35-70 years, were recruited in 10 European countries. At baseline, dietary flavonoid and lignan intakes were estimated using centre-specific validated questionnaires and a food composition database based on the Phenol-Explorer, the UK Food Standards Agency and the US Department of Agriculture databases.

Results: During an average of 11 years of follow-up, 1575 new cases of primary bladder cancer were identified, of which 1425 were UCC (classified into aggressive $(n=430)$ and non-aggressive $(n=413)$ UCC). No association was found between total flavonoid intake and bladder cancer risk. Among flavonoid subclasses, significant inverse associations with bladder cancer risk were found for intakes of flavonol (hazard ratio comparing fifth with first quintile ( $\left.\mathrm{HR}_{\mathrm{Q}_{-}-\mathrm{Q} 1}\right)$ 0.74, 95\% confidence interval (Cl): 0.61-0.91; $P$-trend $=0.009)$ and lignans $\left(\mathrm{HR}_{\mathrm{Q}_{5}-\mathrm{Q} 1} 0.78,95 \% \mathrm{Cl}\right.$ : 0.62-0.96; $P$-trend $\left.=0.046\right)$. Similar results were observed for overall UCC and aggressive UCC, but not for non-aggressive UCC.

Conclusions: Our study suggests an inverse association between the dietary intakes of flavonols and lignans and risk of bladder cancer, particularly aggressive UCC.

Bladder cancer is the sixth most common cancer type and the seventh most common cause of death from cancer in Europe overall, although certain populations are highly affected (Ferlay et al, 2010). Indeed, men are about three times more likely to develop bladder cancer compared with women (Ferlay et al, 2010). Moreover, it is predominantly a disease of high-income countries and overall rates have remained relatively stable over the last decades (Burger et al, 2013).

In 2007, a comprehensive review by the World Cancer Research Fund and the American Institute for Cancer Research concluded that established risk factors for bladder cancer include tobacco consumption, infection with Schistosoma haematobium, and both occupational and environmental exposures to carcinogens such as aromatic amines and polycyclic aromatic hydrocarbons and arsenic in drinking water. In contrast they showed that food, nutrition and physical activity only had modest effects in the development of bladder cancer (World Research Cancer Fund and American Institute for Cancer Research, 2007). Since then, investigations of the European Prospective Investigation into Cancer and Nutrition (EPIC) cohort have found that fruit and vegetable intake measured from dietary questionnaires was not clearly related to bladder cancer (Buchner et al, 2009; Ros et al, 2012), although higher plasma carotenoids concentrations were associated with lower incidence of bladder cancer (Ros et al, 2010); suggesting that specific compounds in fruit and vegetables may have protective associations with bladder cancer risk. In addition, a recent study in the multiethnic cohort has suggested that intake of fruit and vegetables and some related micronutrients such as vitamins $\mathrm{A}, \mathrm{C}, \mathrm{E}$ and carotenoids were inversely associated with bladder cancer risk only in women (Park et al, 2013).

One group of bioactive compounds in fruit and vegetables of growing interest for chronic disease prevention is polyphenols (basically flavonoids, phenolic acids and lignans), which have been shown to have antioxidant, anti-inflammatory and anti-carcinogenic effects in animals and in vitro studies (Yao et al, 2004; Xiao et al, 2011). The biological activity of flavonoids and lignans in the prevention of bladder cancer is plausible considering that most of the flavonoid and lignan metabolites are excreted through urine, exposing the bladder lining to these metabolites (Manach et al, 2005).

To the best of our knowledge, only the Iowa Women's Health Study prospectively assessed the relationship between flavan-3-ol monomer intake (a flavonoid subclass) and bladder cancer risk, and found no association (Arts et al, 2002). Moreover, in a Spanish case-control study, no associations were observed with some individual flavonols and flavones (Garcia et al, 1999). Further epidemiological studies in other large populations (including men) and the assessment of the effect of the other flavonoid subclasses are needed. Therefore, the aim of this study was to investigate the association between the dietary intake of both total and subclasses of flavonoids and lignans and bladder cancer risk in the EPIC study, a large cohort with a high variability in the intake of these compounds (Zamora-Ros et al, 2012, 2013).

\section{MATERIALS AND METHODS}

Study design. The EPIC is an on-going multicentre cohort study designed to examine the association between diet, lifestyle and environmental factors and cancer. The full rationale, methods and design have been described previously (Riboli and Kaaks, 1997; Riboli et al, 2002). Briefly, the EPIC study involves more than half a million men and women from 23 centres in 10 European countries (Denmark, France, Germany, Greece, Italy, the Netherlands, Norway, Spain, Sweden and the United Kingdom). Participants, mostly 
aged between 35 and 70 years, were recruited from primarily the general population during the period 1991-2000 (Riboli and Kaaks, 1997; Riboli et al, 2002). All participants gave written informed consent, and the study was approved by the local ethics committees in the participating countries and the ethical review board of the International Agency for Research on Cancer (IARC).

Case ascertainment and follow-up. Follow-up for end point status was mostly based on population cancer registries, except for France, Germany and Greece, where a combination of methods including health insurance records, cancer and pathology hospital registries, and active follow-up were used. Mortality data were also collected from registries at the regional or national level. The date of the last complete follow-up (recorded in a central database at IARC) ranged from December 2004 to June 2010, depending on the centre.

Bladder cancer cases were coded as C67 following the third edition of the International Classification of Diseases for Oncology (ICD-O-3). Bladder cancer cases with morphology codes 8980 (carcinosarcoma), 9590 (malignant lymphoma), 9671 (malignant lymphoma, lymphoplasmacytic) and tumours with benign behaviours were censored at date of diagnosis. The analyses were also done separately for the urothelial cell carcinomas (UCCs), including urothelial cell papillomas and carcinomas (morphology codes $812-813$ ). The UCCs make up $>90 \%$ of bladder tumours (Allen et al, 2013). The UCC cases were separated into relatively aggressive (high risk of progression) and non-aggressive (low risk of progression) (Kiemeney et al, 2008), as described previously (Ros et al, 2012). Briefly, aggressive UCC cases were defined as stage $\mathrm{T} 1$ and higher or carcinoma in situ (CIS) or World Health Organization (WHO) grade 3, whereas non-aggressive UCC cases were defined as: stage Ta grade 1 or stage Ta grade 2 . Tumour aggressiveness was only classified for UCC cases diagnosed up until 2007, since these have been individually validated by pathology reports, and the remaining tumours were censored at date of diagnosis for stratified analyses.

Participants were excluded from the analyses if they had an extreme energy intake and/or expenditure (participant in the top or the bottom $1 \%$ of the distribution of the ratio of total energy intake to energy requirement) $(n=9600)$ or if information on dietary intake and lifestyle was incomplete $(n=6253)$. Furthermore, 28289 participants were excluded because they had a prevalent cancer at any site at baseline or were lost to follow-up. In this present analysis, 477312 subjects were included.

Dietary and lifestyle assessment. At recruitment, participant's dietary intake in the previous year was estimated using validated and centre-specific questionnaires (Margetts and Pietinen, 1997; Riboli et al, 2002). Dietary questionnaires in most of the centres were quantitative, estimating portion sizes systematically. In Denmark, Norway, Umeå (Sweden) and Naples (Italy), semiquantitative food-frequency questionnaires were administered. In Malmö (Sweden), a modified diet history method was used, combining information from a 7-day diet record, a semiquantitative questionnaire and a $1-\mathrm{h}$ dietary interview. Energy $\left(\mathrm{kcal} \mathrm{day}^{-1}\right)$ and ethanol $\left(\mathrm{g} \mathrm{day}^{-1}\right)$ intakes were estimated using the EPIC Nutrient Database (Slimani et al, 2007). Lifestyle questionnaires included questions on education, medical history, lifetime history of consumption of tobacco and physical activity (Riboli et al, 2002; Wareham et al, 2003). Height and weight at baseline were measured in most of the centres, except for Oxford (UK), France and Norway where anthropometric measures at baseline were selfreported. BMI was calculated as weight in kilograms divided by squared height in metres $\left(\mathrm{kg} \mathrm{m}^{-2}\right)$.

Dietary intake of total and subclasses of flavonoids (flavanols (including flavan-3-ol monomers, proanthocyanidins, theaflavins), anthocyanidins, flavonols, flavanones, flavones, and isoflavones) and total lignans were estimated using our own database (ZamoraRos et al, 2012, 2013), which combines food composition data from the USDA databases (U.S.Departament of Agriculture, 2004, 2007, 2008), Phenol-Explorer (Neveu et al, 2010) and the UK Food Standards Agency database (Ward et al, 2010). Moreover, our food composition database was expanded by using retention factors (except for isoflavones and lignans) (Crozier et al, 1997; U.S.Departament of Agriculture, 2008), developing recipes, and estimating missing values based on similar foods (by botanical family and plant part). Data on flavonoids and lignans are expressed as aglycone equivalents, after conversing flavonoid glycosides into aglycone contents using their respective molecular weights. The final database contains 1877 food items (10\% have missing values) and includes raw foods, cooked foods and recipes.

Statistical analysis. Flavonoid and lignan intake was assessed by the mean and its s.d., as well as the median and the 5th and 95th centiles (P5th, P95th) because the data were skewed to the right. The distribution of the population's main characteristics according to quintiles of total flavonoid intakes were examined using twosided $\chi^{2}$ and Kruskal-Wallis tests, as appropriate. The relationships between dietary intakes of flavonoids and lignans and bladder cancer risk were assessed by estimating the hazard ratio (HR) and its $95 \%$ confidence interval (CI) in Cox regression models. Tests and graphs based on Schoenfeld residuals were used to assess the proportional hazards assumption, which was satisfied. Total and subclasses of flavonoids and lignans were categorised using cohortwide quintiles. Tests for linear trend were performed by assigning the medians of each quintile as scores. Intakes were also analysed continuously after a $\log _{2}$ transformation that indicates a reduction of bladder cancer risk for doubling flavonoid and lignan intakes. Hazard ratios were estimated using the following modelling strategy; age was used as the underlying time scale, with entry time defined as the participant's age at baseline and exit time as age at cancer diagnosis (for cases) or censoring (for at-risk participants), which was age at death or end of follow-up. Crude models were stratified by centre, sex and age at baseline (1-year intervals). Multivariable model 1 was additionally adjusted for total energy intake ( $\mathrm{kcal} \mathrm{day}^{-1}$, continuous variable) and smoking status and intensity (never, former quit $<11$ years, former quit 11-20 years, former quit $>20$ years, current $<15$ cigarettes day $^{-1}$, current 15-25 cigarettes day ${ }^{-1}$, current $>25$ cigarettes day $^{-1}$, current occasional smoker of pipe, cigarettes or other types of tobacco, current/ former smokers with unknown value of intensity or time since cessation and not specified). Other potential confounders were additionally adjusted for in multivariable model 2, such as BMI $\left(\mathrm{kg} \mathrm{m}^{-2}\right)$, physical activity (inactive, moderately inactive, moderately active, active and not specified), highest educational level (none, primary school, technical/professional school, secondary school, university or higher and not specified) and alcohol intake $\left(\mathrm{g} \mathrm{day}^{-1}\right)$. Any of these variables did not change effect estimates $>10 \%$, and therefore in the results we only show the multivariable model 1 because the results in both models were almost identical. The interactions between sex, BMI status $(<25 ; 25-29.9 ; \geqslant 30 \mathrm{~kg}$ $\mathrm{m}^{-2}$ ) or tobacco status (never, former and current smokers) and total and subclasses of flavonoid and lignan intakes were tested using likelihood ratio tests based on the models with and without the interaction terms. Sensitivity analyses were performed after exclusion of 181 cases who were diagnosed during the first 2 years of follow-up. $P$-values $<0.05 \quad$ (two-tailed) were considered significant. All analyses were conducted using SAS version 9.1 software (SAS Institute, Inc., Cary, NC, USA).

\section{RESULTS}

Among 477312 (29.8\% men) participants included in this study, with a mean follow-up of 11.0 years, 1575 (70.3\% men) incident primary bladder cancer cases were diagnosed, of which 1425 were 
identified as UCC (including 430 aggressive, 413 non-aggressive and 52 unknown tumour aggressiveness UCC cases, and 530 unclassified cases; Table 1). The distribution of total and subclasses of flavonoid and lignan intakes and their main food sources is shown in the Table 2. The median intake of total flavonoids and total lignans were $332.4 \mathrm{mg} \mathrm{day}^{-1}$ and $1.3 \mathrm{mg} \mathrm{day}^{-1}$, respectively. Descriptive characteristics of the population by quintiles of total flavonoid intake are shown in Table 3 . There was a slightly greater percentage of men and higher age at recruitment in the fifth quintile compared with the first one. In addition, participants in the fifth quintile had the lowest BMI, reported higher total energy and alcohol. Furthermore, participants in the top quintile tended to smoke less, have a higher educational level and be more physically active compared with those in the bottom quintile.

Statistically significant inverse associations were found in the crude models between bladder cancer risk and the intake of total flavonoids, flavanols, flavan-3-ol monomers, proanthocyanidins, theaflavins, flavonols, flavones and lignans; although in multivariable models, only the intakes of flavonols $\left(\mathrm{HR}_{\mathrm{Q} 5-\mathrm{Q} 1} 0.75,95 \%\right.$ CI: $0.61-0.91 ; P$-trend $=0.009)$ and lignans $\left(\mathrm{HR}_{\mathrm{Q} 5-\mathrm{Q} 1} 0.78,95 \%\right.$ CI: $0.62-0.96$; $P$-trend $=0.042)$ maintained the significant inverse association with bladder cancer risk (Table 4).

Similar relationships were observed for UCC (Table 4), where only flavonol intake was inversely associated with UCC risk $\left(\mathrm{HR}_{\mathrm{Q} 5-\mathrm{Q} 1}\right.$ 0.76, 95\% CI: 0.62-0.94; $P$-trend $\left.=0.022\right)$ and lignan intake tended to be inversely related to UCC risk $\left(\mathrm{HR}_{\mathrm{Q} 5-\mathrm{Q} 1} 0.79\right.$, 95\% CI: $0.63-1.00 ; P$-trend $=0.090$ ) in the multivariable model. According to tumour stage and grade, flavonol $\left(\mathrm{HR}_{\mathrm{Q} 5-\mathrm{Q} 1} 0.64,95 \%\right.$ CI: 0.44-0.95; $P$-trend $=0.020)$ and lignan $\left(\mathrm{HR}_{\mathrm{Q} 5-\mathrm{Q} 1} 0.59,95 \% \mathrm{CI}\right.$ : $0.39-0.89 ; \quad P$-trend $=0.035)$ intakes were inversely related to aggressive UCC, but not to non-aggressive UCC (Table 4).

There was no evidence that the relation between total flavonoid and lignan intake and bladder cancer was modified by sex $(P$ for interaction $=0.19$ and 0.23 ) or BMI $(P$ for interaction $=0.52$ and $0.30)$. Although the interactions with tobacco consumption were not significant for the intake of total flavonoids and lignans $(P$ for interaction $=0.47$ and 0.90 , respectively), separate analyses were presented for smoking status because it is a major risk factor of bladder cancer (Supplementary Table 1). Similar results were observed between the intake of total and subclasses of flavonoids and lignans and bladder cancer risk (and subtypes) in never and current smokers.

In the sensitivity analysis, similar associations between the intake of flavonols $\left(\mathrm{HR}_{\mathrm{Q} 5-\mathrm{Q} 1} \quad 0.69,95 \%\right.$ CI: $0.56-0.86$;
$P$-trend $=0.004)$ and lignans $\left(\mathrm{HR}_{\mathrm{Q} 5-\mathrm{Q} 1} 0.76,95 \% \mathrm{CI}\right.$ : $0.60-0.97$; $P$-trend $=0.055)$ and incidence of overall bladder cancer were observed after the exclusion of bladder cancer cases diagnosed within the first 2 years of follow-up.

\section{DISCUSSION}

The results in this large cohort of participants from 10 Western European countries suggest that higher dietary intakes of flavonols and lignans may be associated with an approximately $25 \%$ lower bladder cancer risk. These protective associations were also observed for UCC, in particular the aggressive tumours. No associations were observed with either total or other flavonoid subclasses.

To the best of our knowledge, there is only one other prospective epidemiological study on flavonoids and bladder cancer, which reported no association between flavan-3-ol monomers and bladder cancer risk in the Iowa Women's Health Study, a postmenopausal women cohort from the United States (Arts et al, 2002). Our finding provides further evidence for the absence of any strong association between flavan-3-ol monomers, one of the most abundant flavonoid subclasses, primarily found in tea, and bladder cancer risk in a cohort that contains more cancer cases (1575 vs 103) and includes men, who are at greater risk of developing bladder cancer (70.3\% of bladder cases). Conversely to our results, in a Spanish case-control study, no association with some individual flavonols and flavones was observed, but the food composition table used in this study was quite old and therefore, very limited (Garcia et al, 1999). In relation to other cancers of the urinary tract, intake of flavonols and particularly quercetin was inversely associated with renal cell carcinoma risk in the AlphaTocopherol Beta-Carotene Cancer Prevention Study (Wilson et al, 2009) and in an Italian case-control study (Bosetti et al, 2007), but not total flavonoids. However, to our knowledge, there are no studies assessing the relationship between lignan intake and risk of urinary tract cancers.

A randomised controlled trial, with an intervention group that followed a month long diet rich in flavonoids from a typical Mediterranean diet and green tea, showed a strong correlation between urinary phenolics and an anti-mutagenicity activity, indicated by an inhibition effect of urinary extracts on Salmonella typhimurium mutations induced by MeIQx (as model substrate for cytochrome P4501A2) (Malaveille et al, 2004). From the same

Table 1. Distribution of participants and bladder cancer and UCC cases according to tumour aggressiveness during 11 years of follow-up in the 10 countries participating in the EPIC study

\begin{tabular}{|c|c|c|c|c|c|c|}
\hline Country & All & Person-years & Bladder cancer cases & UCC cases & Aggressive UCC & Non-aggressive UCC \\
\hline Denmark & 55016 & 601466 & 303 & 284 & 112 & 80 \\
\hline France & 67385 & 699360 & 31 & 24 & 13 & 6 \\
\hline Germany & 48583 & 480614 & 199 & 171 & 46 & 40 \\
\hline Greece & 26032 & 247711 & 45 & 25 & 5 & 6 \\
\hline Italy & 44541 & 500407 & 183 & 143 & 45 & 42 \\
\hline Norway & 35169 & 342279 & 24 & 24 & 8 & 2 \\
\hline Spain & 40002 & 482582 & 146 & 138 & 24 & 61 \\
\hline Sweden & 48684 & 638931 & 289 & 281 & 90 & 80 \\
\hline The Netherlands & 36505 & 431252 & 104 & 101 & 30 & 29 \\
\hline United Kingdom & 75395 & 838397 & 251 & 234 & 57 & 67 \\
\hline TOTAL & 477312 & 5262998 & 1575 & 1425 & 430 & 413 \\
\hline
\end{tabular}


Table 2. Dietary intake of flavonoids and lignans (in $\mathrm{mg} \mathrm{day}^{-1}$ ) and their main food sources in the EPIC study

\begin{tabular}{|l|r|r|r|c|l|l|}
\hline & Mean & \multicolumn{1}{|c|}{ S.d. } & Median & Percentile 5 & Percentile 95 & Food sources \\
\hline Total flavonoids & 437.2 & 335.0 & 332.4 & 90.8 & 1137.7 & Fruits (40\%), tea (19\%), wine (12\%), fruit juices (6\%) \\
\hline Flavanols & 355.3 & 309.2 & 248.9 & 58.9 & 1013.4 & Tea (44\%), fruit (29\%), wine (9\%), chocolates (4\%) \\
\hline Flavan-3-ols monomers & 176.6 & 253.5 & 46.4 & 9.4 & 731.5 & Tea (84\%), fruits (6\%), wine (3\%), chocolates (2\%) \\
Proanthocyanidins & 172.8 & 123.9 & 148.3 & 41.2 & 388.2 & Fruits (53\%), wine (14\%), chocolates (6\%), tea (4\%) \\
Theaflavins & 5.8 & 9.8 & 0.4 & 0 & 27.2 & Tea (100\%) \\
\hline Anthocyanidins & 28.3 & 22.5 & 22.5 & 5.4 & 70.4 & Fruits (52\%), wine (21\%), vegetables (8\%), fruit juices (7\%) \\
\hline Flavonols & 27.2 & 17.3 & 22.4 & 8 & 61.8 & Tea (26\%), vegetables (23\%), fruits (13\%), soups (12\%) \\
\hline Flavanones & 21.9 & 22.8 & 15.7 & 1.7 & 64.8 & Fruits (50\%), fruit juices (41\%), wine (5\%), vegetables (1\%) \\
\hline Flavones & 3.5 & 4.1 & 2.5 & 0.4 & 9.8 & Herbal tea (30\%), wine (18\%), fruits (16\%), vegetables (15\%) \\
\hline Isoflavones & 1.5 & 4.9 & 0.5 & 0.1 & 4.4 & Soya products (40\%), cakes (18\%), cereals (11\%), coffee (8\%) \\
\hline Lignans & 1.5 & 0.8 & 1.3 & 0.6 & 2.9 & Vegetables (24\%), fruits (17\%), cereals (16\%), tea (10\%) \\
\hline Abbreviation: EPIC=European Prospective Investigation into Cancer and Nutrition study. & \\
\hline
\end{tabular}

Table 3. Baseline characteristics of the participants in the EPIC study according to quintiles of total flavonoids intake

Total flavonoid intake

\begin{tabular}{|c|c|c|c|c|c|c|c|}
\hline Characteristics & $\begin{array}{c}\text { All } \\
(n=477312)\end{array}$ & $\begin{array}{c}\text { Q1 } \\
(n=95462)\end{array}$ & $\begin{array}{c}\text { Q2 } \\
(n=95463)\end{array}$ & $\begin{array}{c}\text { Q3 } \\
(n=95462)\end{array}$ & $\begin{array}{c}\text { Q4 } \\
(n=95463)\end{array}$ & $\begin{array}{c}\text { Q5 } \\
(n=95462)\end{array}$ & $P$-value \\
\hline Median flavonoid intake $\left(\mathrm{mg} \mathrm{day}^{-1}\right)$ & 332.4 & 123.9 & 225.4 & 332.4 & 514.6 & 933.4 & \\
\hline Sex, men (\%) & 29.8 & 29.7 & 29.8 & 29.1 & 29.5 & 30.9 & $<0.001$ \\
\hline Age at enrolment (years) ${ }^{a}$ & $51.2(9.9)$ & $50.9(9.1)$ & $51.2(9.6)$ & $50.9(9.6)$ & $51.3(9.9)$ & $51.8(11.3)$ & $<0.001$ \\
\hline BMI $\left(\mathrm{kg} \mathrm{m}^{-2}\right)^{\mathrm{a}}$ & $25.4(4.3)$ & $25.7(4.4)$ & $25.8(4.4)$ & $25.6(4.3)$ & $25.3(4.2)$ & $24.8(4.0)$ & $<0.001$ \\
\hline Energy intake $\left(\mathrm{kcal} \mathrm{day}^{-1}\right)^{\mathbf{a}}$ & 2074 (619) & 1791 (545) & 2010 (569) & 2142 (598) & 2211 (638) & 2217 (635) & $<0.001$ \\
\hline Alcohol intake $\left(\mathrm{g} \mathrm{day}^{-1}\right)^{\mathrm{a}}$ & $11.9(17.1)$ & $7.5(12.2)$ & $10.6(15.4)$ & $12.4(16.9)$ & $14.5(18.7)$ & $14.7(20.1)$ & $<0.001$ \\
\hline Educational level (\%) & & & & & & & $<0.001$ \\
\hline $\begin{array}{l}\text { No formal education } \\
\text { Primary school } \\
\text { Technical/professional school } \\
\text { Secondary school } \\
\text { University degree } \\
\text { Not specified }\end{array}$ & $\begin{array}{r}4.4 \\
25.6 \\
22.3 \\
20.4 \\
23.8 \\
3.5\end{array}$ & $\begin{array}{r}5.0 \\
33.4 \\
27.2 \\
18.3 \\
15.0 \\
1.1\end{array}$ & $\begin{array}{r}5.9 \\
29.2 \\
22.1 \\
20.0 \\
21.1 \\
1.7\end{array}$ & $\begin{array}{r}5.5 \\
26.3 \\
19.0 \\
22.8 \\
24.3 \\
2.1\end{array}$ & $\begin{array}{r}3.9 \\
23.0 \\
20.0 \\
22.4 \\
26.9 \\
3.8\end{array}$ & $\begin{array}{r}1.7 \\
16.1 \\
23.1 \\
18.6 \\
31.6 \\
8.9\end{array}$ & \\
\hline Smoking status and intensity (\%) & & & & & & & $<0.001$ \\
\hline $\begin{array}{l}\text { Never smoker } \\
\text { Current, }<15 \text { cigarretes day }^{-1} \\
\text { Current, } 15-25 \text { cigarretes day }^{-1} \\
\text { Current, } 25 \text { cigarretes day }^{-1} \\
\text { Former, from } \leqslant 10 \text { years } \\
\text { Former, from } 11 \text { to } 20 \text { years } \\
\text { Former, from }>20 \text { years } \\
\text { Current, pipe/cigar/occas } \\
\text { Current/former, missing } \\
\text { Not specified }\end{array}$ & $\begin{array}{r}43.0 \\
11.6 \\
6.3 \\
1.8 \\
9.6 \\
8.2 \\
7.9 \\
8.4 \\
1.6 \\
1.6\end{array}$ & $\begin{array}{r}36.4 \\
17.0 \\
11.2 \\
2.9 \\
9.9 \\
6.8 \\
6.6 \\
5.3 \\
1.7 \\
2.2\end{array}$ & $\begin{array}{r}43.0 \\
12.7 \\
6.9 \\
2.3 \\
9.5 \\
7.7 \\
7.0 \\
7.8 \\
1.5 \\
1.6\end{array}$ & $\begin{array}{r}44.3 \\
11.1 \\
5.5 \\
1.8 \\
9.4 \\
8.1 \\
6.8 \\
10.2 \\
1.3 \\
1.5\end{array}$ & $\begin{array}{r}44.6 \\
9.4 \\
4.4 \\
1.3 \\
9.7 \\
9.0 \\
8.2 \\
10.6 \\
1.5 \\
1.3 \\
\end{array}$ & $\begin{array}{r}46.8 \\
7.8 \\
3.2 \\
0.8 \\
9.3 \\
9.2 \\
10.9 \\
8.1 \\
2.1 \\
1.8\end{array}$ & \\
\hline Physical activity (\%) & & & & & & & $<0.001$ \\
\hline $\begin{array}{l}\text { Inactive } \\
\text { Moderately inactive } \\
\text { Moderately active } \\
\text { Active } \\
\text { Not specified } \\
\end{array}$ & $\begin{array}{r}20.7 \\
31.3 \\
22.2 \\
17.0 \\
8.8 \\
\end{array}$ & $\begin{array}{l}20.0 \\
24.8 \\
16.4 \\
12.4 \\
26.4 \\
\end{array}$ & $\begin{array}{r}22.5 \\
31.2 \\
21.1 \\
15.4 \\
9.8 \\
\end{array}$ & $\begin{array}{r}22.0 \\
33.7 \\
23.5 \\
16.9 \\
3.9 \\
\end{array}$ & $\begin{array}{r}19.5 \\
34.2 \\
24.9 \\
19.3 \\
2.1 \\
\end{array}$ & $\begin{array}{r}19.3 \\
32.8 \\
25.2 \\
20.9 \\
1.8 \\
\end{array}$ & \\
\hline
\end{tabular}




\begin{tabular}{|c|c|c|c|c|c|c|c|c|c|c|}
\hline \multirow{3}{*}{ 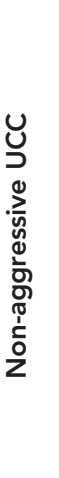 } & 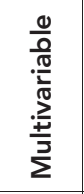 & $\begin{array}{l}\widehat{u} \\
\stackrel{\circ}{\stackrel{\circ}{0}} \\
\stackrel{\alpha}{I}\end{array}$ & & 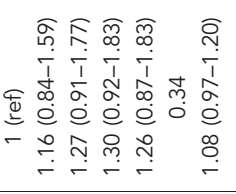 & & 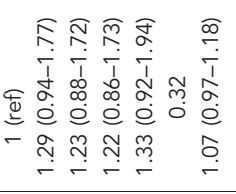 & & 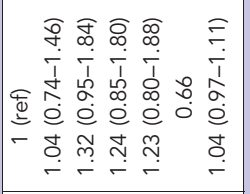 & & 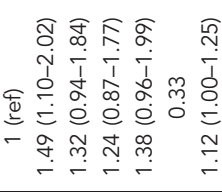 \\
\hline & 造 & 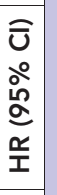 & & 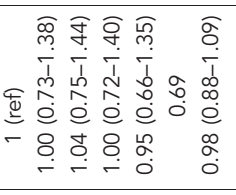 & & 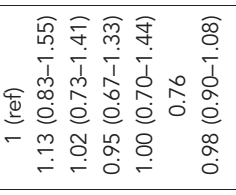 & & 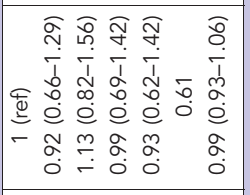 & & 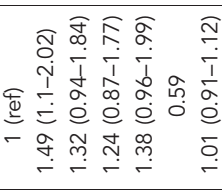 \\
\hline & 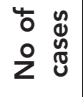 & & & $\bar{\infty} \ltimes \stackrel{\infty}{\infty} \infty \bar{\sigma}$ & & 요 $\curvearrowright \infty$ & & J & & œ \\
\hline \multirow{3}{*}{ 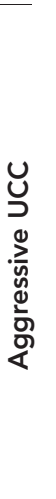 } & 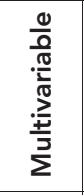 & 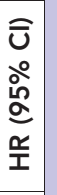 & & 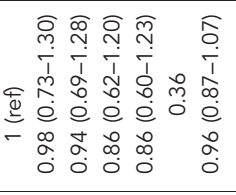 & & 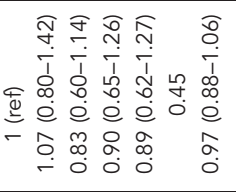 & & 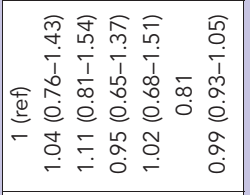 & & 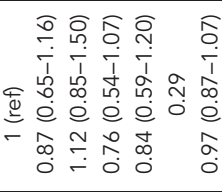 \\
\hline & $\begin{array}{l}\frac{0}{0} \\
\frac{0}{2} \\
0\end{array}$ & 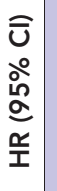 & & 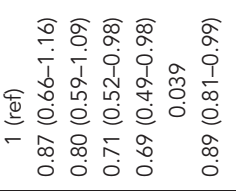 & & 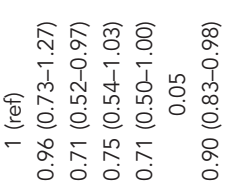 & & 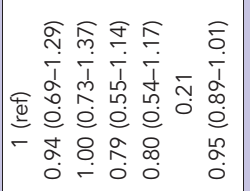 & & 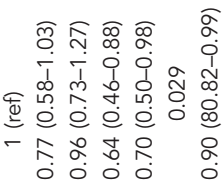 \\
\hline & 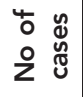 & & & ó $\sigma \stackrel{\infty}{\wedge} \cong \infty$ & & 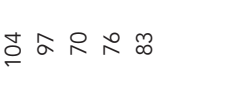 & & ㅇㅇㅇㅇㅇㅇ & & 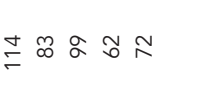 \\
\hline \multirow{3}{*}{ ư } & 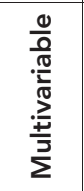 & 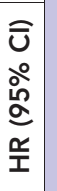 & & 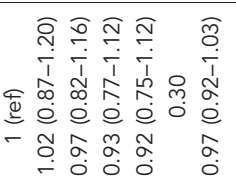 & & 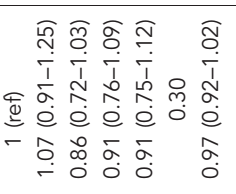 & & 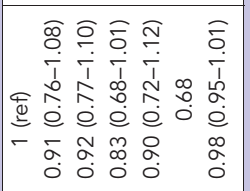 & & 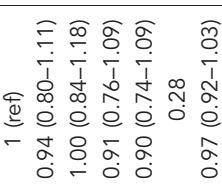 \\
\hline & $\frac{0}{0}$ & 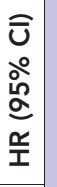 & & 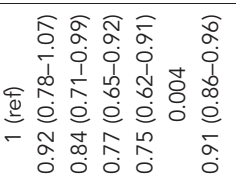 & & 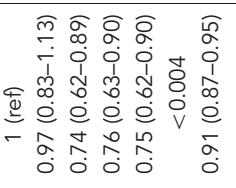 & & 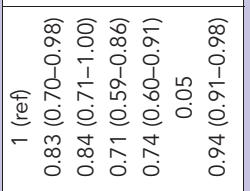 & & 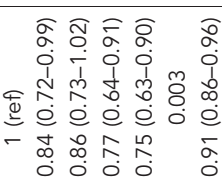 \\
\hline & 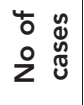 & & & 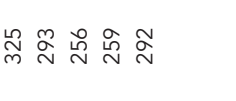 & & $\frac{\infty}{m} \stackrel{\infty}{\circ} \stackrel{\infty}{\sim} \stackrel{+}{\sim} \stackrel{\alpha}{\stackrel{\alpha}{N}}$ & & 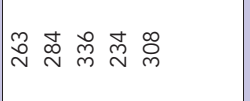 & & 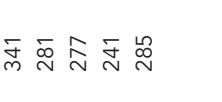 \\
\hline \multirow{5}{*}{ 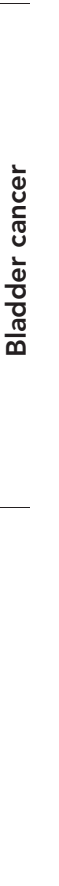 } & 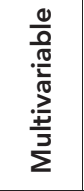 & 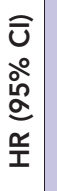 & & 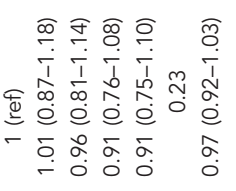 & & 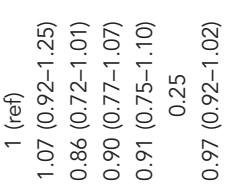 & & 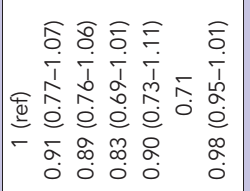 & & 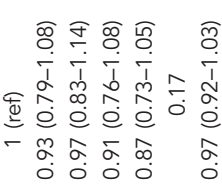 \\
\hline & 造 & 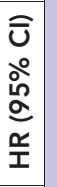 & & 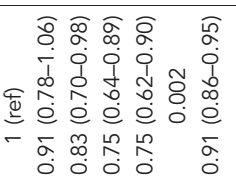 & & 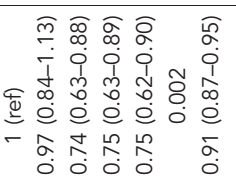 & & 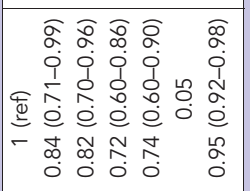 & & 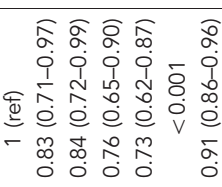 \\
\hline & 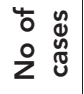 & & & 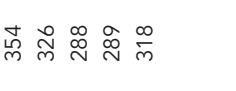 & & 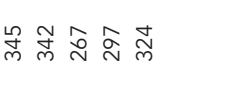 & & 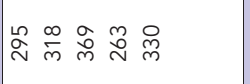 & & 尺্লি \\
\hline & & 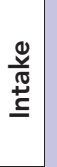 & & 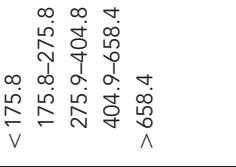 & & 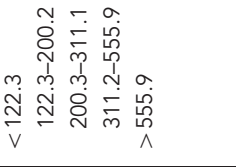 & 峁 & 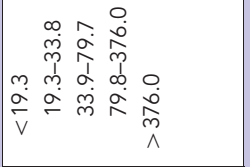 & & 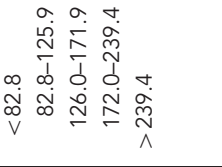 \\
\hline & & & 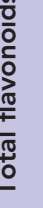 & 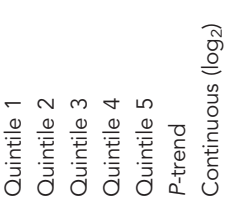 & $\begin{array}{l}\frac{n}{0} \\
\frac{\Gamma}{\pi} \\
\frac{\pi}{14}\end{array}$ & 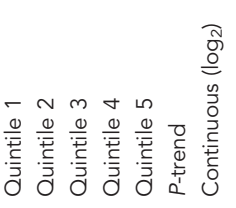 & 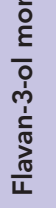 & 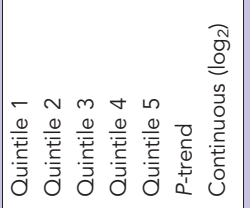 & 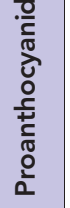 & 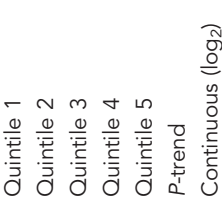 \\
\hline
\end{tabular}




\begin{tabular}{|c|c|c|c|c|c|c|c|c|c|c|}
\hline \multirow{3}{*}{ 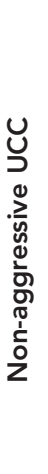 } & $\begin{array}{l}\frac{0}{0} \\
\frac{0}{\frac{\pi}{2}} \\
\frac{20}{\frac{10}{2}} \\
\frac{1}{2}\end{array}$ & 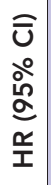 & & 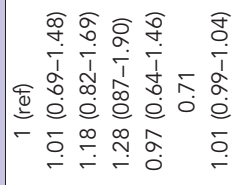 & & 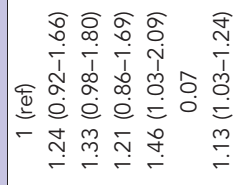 & & 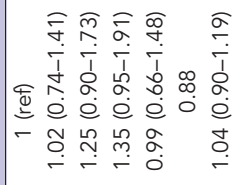 & & 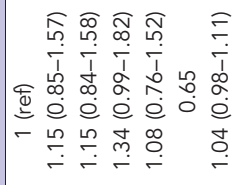 \\
\hline & $\begin{array}{l}\frac{0}{0} \\
\frac{1}{2}\end{array}$ & 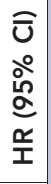 & & 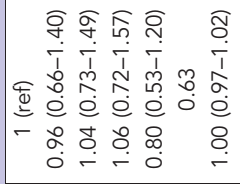 & & 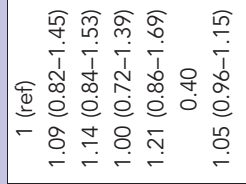 & & 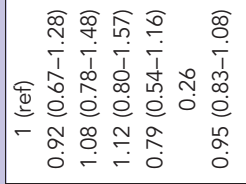 & & 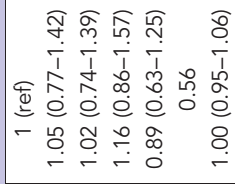 \\
\hline & 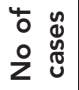 & & & 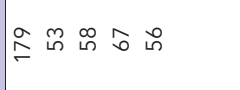 & & œ ০ ฌ & & ネミよミ & & 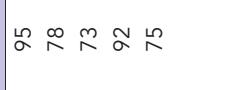 \\
\hline \multirow{3}{*}{ 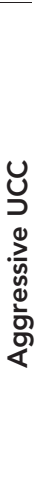 } & 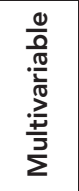 & 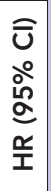 & & 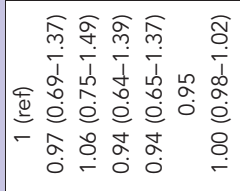 & & 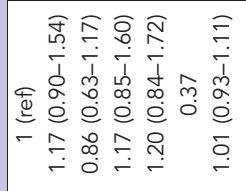 & & 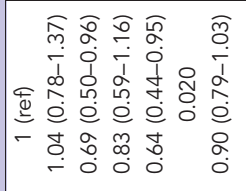 & & 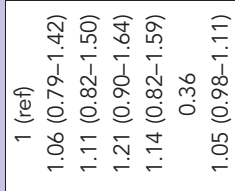 \\
\hline & $\frac{0}{\frac{0}{2}}$ & 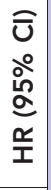 & & 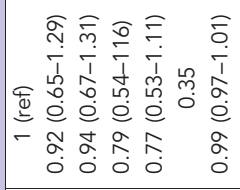 & & 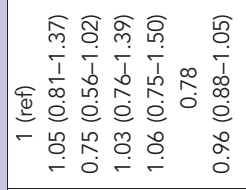 & & 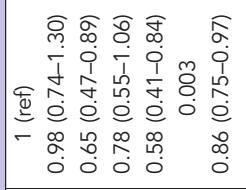 & & 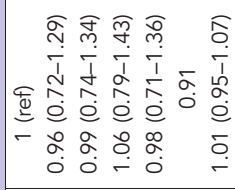 \\
\hline & 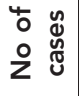 & & & 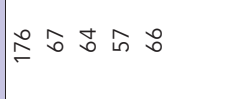 & & 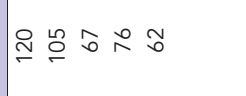 & & ¿용 & & $\varnothing \underset{-\infty}{\infty} \infty \stackrel{\infty}{\wedge}$ \\
\hline \multirow{3}{*}{ ني } & $\begin{array}{l}\frac{0}{0} \\
\frac{0}{\frac{0}{2}} \\
\frac{2}{\pi} \\
\frac{2}{5} \\
\sum\end{array}$ & 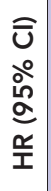 & & 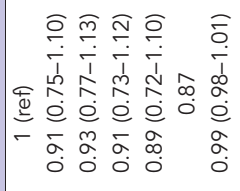 & & 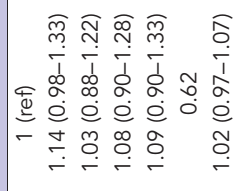 & & 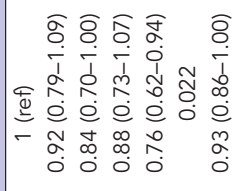 & & 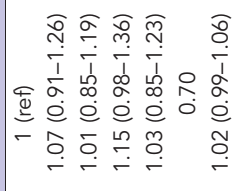 \\
\hline & $\begin{array}{l}\frac{0}{0} \\
\text { ㄹํㄴ }\end{array}$ & 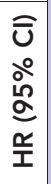 & & 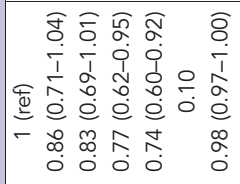 & & 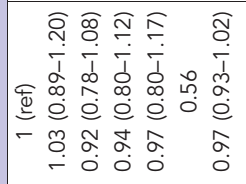 & & 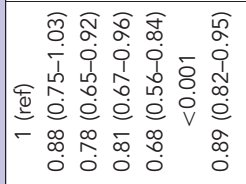 & & 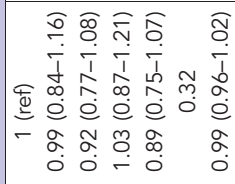 \\
\hline & 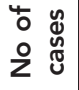 & & & $\stackrel{n}{\sigma} \stackrel{m}{\stackrel{\sigma}{\sigma}} \stackrel{m}{\sim} \stackrel{m}{\sim}$ & & 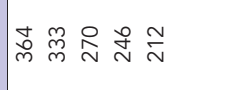 & & 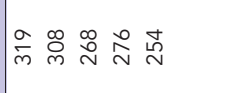 & & 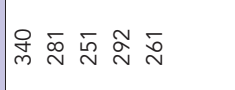 \\
\hline \multirow{5}{*}{ 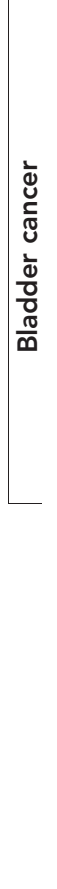 } & $\begin{array}{l}\frac{0}{0} \\
\frac{0}{0} \\
\frac{\pi}{2} \\
\frac{0}{10} \\
\frac{1}{5}\end{array}$ & 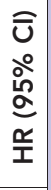 & & 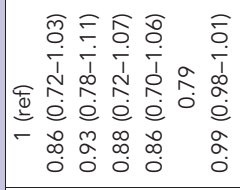 & & 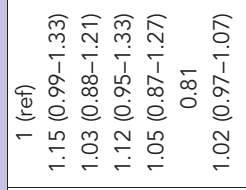 & & 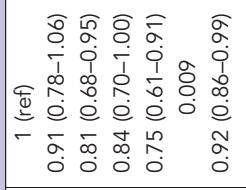 & & 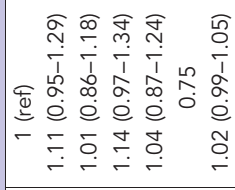 \\
\hline & $\frac{\dddot{0}}{\frac{0}{2}}$ & 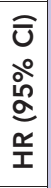 & & 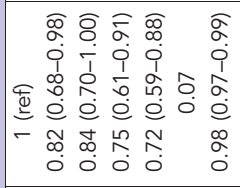 & & 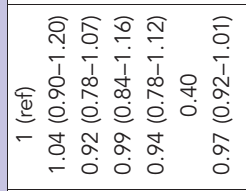 & & 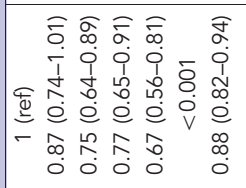 & & 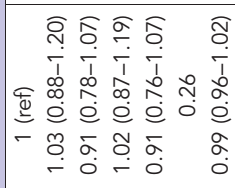 \\
\hline & 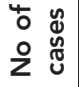 & & & 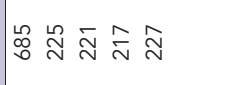 & & 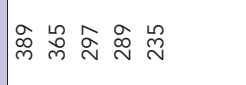 & & 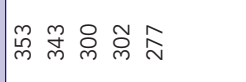 & & 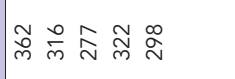 \\
\hline & & 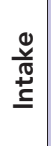 & & 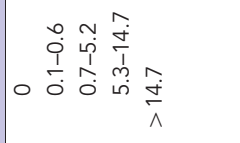 & & 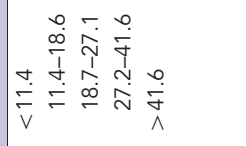 & & 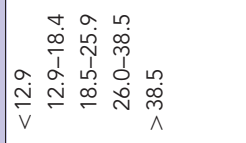 & & 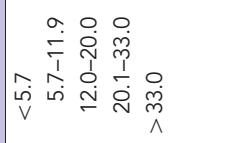 \\
\hline & & & 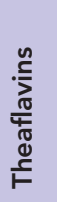 & 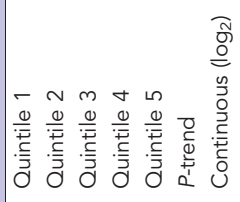 & 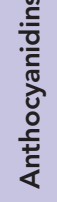 & 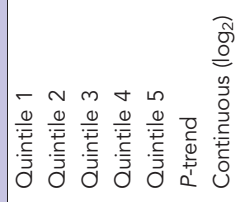 & $\begin{array}{l}\frac{n}{0} \\
\frac{0}{0} \\
\frac{\pi}{4}\end{array}$ & 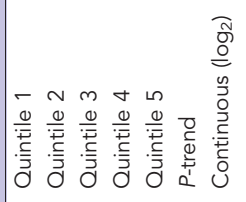 & $\begin{array}{l}\stackrel{n}{0} \\
\stackrel{0}{0} \\
\stackrel{0}{\pi} \\
\frac{\pi}{4} \\
\frac{2}{4}\end{array}$ & 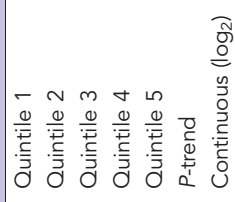 \\
\hline
\end{tabular}




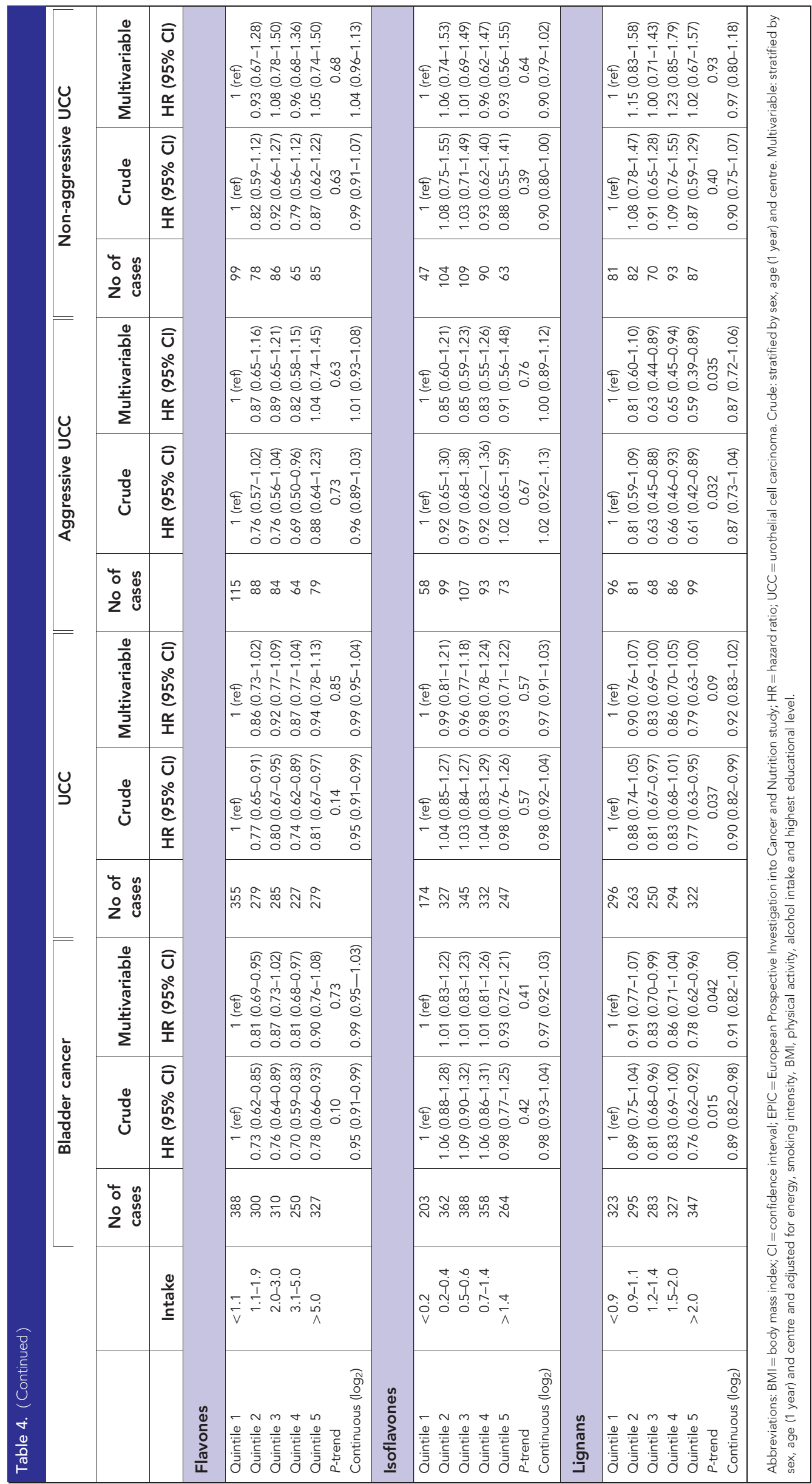


study, an increase in flavonoid intake was associated with a decrease in DNA adducts, suggesting that a diet rich in flavonoids may reduce genotoxicity in the human urinary bladder (Talaska et al, 2006).

Epidemiological studies have suggested that a Mediterranean diet reduces the risk of cancers at different organ sites (World Research Cancer Fund and American Institute for Cancer Research, 2007); although, in a recent study in the EPIC cohort, no significant association was observed between adherence to the Mediterranean diet and risk of bladder cancer in the overall cohort (Buckland et al, 2014). However, in current smokers, a protective association was suggested. A high concentration of flavonoids and other polyphenols has been found in fruits and vegetables, red wine, and other elements of the Mediterranean diet (Perez-Jimenez et al, 2010). Despite this, there is inconsistent evidence on the role of total fruit and vegetable intakes measured by dietary questionnaires on bladder cancer risk (Sacerdote et al, 2007; Buchner et al, 2009; Ros et al, 2012; Park et al, 2013). However, intake of some vegetables subgroups was inversely associated with bladder cancer risk, such as root vegetables in previous EPIC studies (Buchner et al, 2009), particularly in aggressive UCC (Ros et al, 2012), and yellow-orange vegetables in the Multiethnic Cohort study (Park et al, 2013). It is worth bearing in mind that vegetables were the main food source of flavonols and lignans in this study. In addition, plasma concentrations of carotenoids (a nutritional biomarker of fruit and vegetable consumption and which are not limited by measurement errors inherent in dietary questionnaires) were inversely associated with bladder cancer risk (Ros et al, 2010). Therefore, several authors suggested that polyphenols and other antioxidant micronutrients may be, at least in part, responsible for the protective effect of the Mediterranean diet on health (Pelucchi et al, 2009; Giacosa et al, 2013).

A beverage that is not typical of the Mediterranean diet but has been related to bladder cancer protection and is rich in flavonoids is green tea (Wang et al, 2013). Green tea polyphenols have been shown to inhibit nitrosamine-mediated carcinogenesis in in vitro bladder models (Chung et al, 1993). Human studies have confirmed that green tea polyphenols interfere with carcinogenesis by reducing nitrosation and chromosome damage (Steele et al, 2000). Green tea is not widely consumed in Europe, and therefore was not in this study. Black tea is one of the main food sources of flavonols, but it is also the main source of other flavonoid subclasses (such as flavan-3-ol monomers and theaflavins) that were not related to bladder cancer in the present study. Therefore, our results suggest that flavonoids from black tea are not related to bladder carcinogenesis, as concluded by a previous meta-analysis (Wang et al, 2013).

In general, flavonoids and lignans can have a protective effect on bladder cancer through inhibition of phase I and induction of phase II enzymes. This protection may arise because of multiple mechanisms of action, further complexified by the synergistic behaviour of several compounds (Russo et al, 2012). In fact, in subjects with higher consumption of foods rich in flavonoids, a general upregulation of DNA repair genes has been observed, and an inverse correlation with gene expression levels, which may suggest a flavonoid-mediated reduction of DNA damage (Guarrera et al, 2007). However, other explanations are also plausible, three of which are: (i) flavonoids interfere with DNA repair; (ii) flavonoids can have a more complex interaction with DNA damage, not based on repair induction; and (iii) flavonoids can interfere with the induction/selection of chromosome instability or bulky DNA adducts by carcinogens.

In particular, individual flavonols have reduced proliferation of bladder cancer cells in in vitro models. Kaempferol (Xie et al, 2013) and myricetin (Sun et al, 2012) induced apoptosis in a dosedependent manner by increasing the cleavage of caspase-3. Moreover, myricetin reduced the migration of human bladder cancer T24 cells and the phosphorylation of Akt and MMP-9 expression; whereas the phosphorylation of p38 MAPK was enhanced (Sun et al, 2012). In addition, quercetin reduced cancer progression by altering the extracellular catabolism of nucleotides in T24 cells (Rockenbach et al, 2013). No data are available on the effects of lignans upon bladder cancer cells.

In terms of our study limitations, first, changes in lifestyle could not be taken into account as we only collected baseline questionnaires of diet and other lifestyle variables. Second, measurement error in collecting self-reported dietary intake is inevitable, although centre-specific validated questionnaires (Riboli and Kaaks, 1997; Riboli et al, 2002) were used. Furthermore, flavonoid and lignan intakes are likely to be underestimated as the flavonoid database was incomplete (although an extensive common database was used) (Zamora-Ros et al, 2012, 2013) and herb/plant supplement intakes were omitted in these analyses (up to $5 \%$ in Denmark, the highest consumer country) (Skeie et al, 2009). This misclassification is likely to be random and therefore any association between intake and disease risk is likely underestimated. Nutritional biomarkers could be used to reduce this exposure misclassification (Zamora-Ros et al, 2014). Third, the association between dietary flavonoids and lignans with bladder cancer risk may be susceptible to confounding because high flavonoid and lignan intake reflects a healthier lifestyle. In our models, we have adjusted for other determinants of healthy lifestyle, however, possible residual confounding, especially due to smoking, cannot be excluded. Despite of that, similar risk estimates were observed in never and current smokers. Finally, we realise that our study is prone to the well-known drawback of multiple comparisons. The strengths of our multicentre study include its prospective and population-based design, detailed information on diet, large heterogeneity in flavonoid and lignan intake and a large sample of bladder cancer cases, with validated information on tumour aggressiveness.

In conclusion, this large prospective study conducted in 10 European countries suggests inverse associations between dietary intakes of flavonols and lignans and risk of bladder cancer, in particular aggressive tumours of UCC. No associations were observed with either total or other flavonoid subclasses.

\section{ACKNOWLEDGEMENTS}

This work was supported by the European Commission: Public Health and Consumer Protection Directorate 1993 to 2004; Research Directorate-General 2005; Ligue contre le Cancer, Institut Gustave Roussy, Mutuelle Générale de l'Education Nationale, Institut National de la Santé et de la Recherche Médicale (INSERM) (France); German Cancer Aid; German Cancer Research Center; German Federal Ministry of Education and Research; Danish Cancer Society; Health Research Fund (FIS) of the Spanish Ministry of Health (RTICC DR06/0020/0091); the participating regional governments from Asturias, Andalucía, Murcia (no. 6236), Navarra and Vasco Country and the Catalan Institute of Oncology of Spain; Cancer Research UK and the Medical Research Council (United Kingdom); the Hellenic Health Foundation; Italian Association for Research on Cancer; Compagnia San Paolo, Italy; Dutch Ministry of Public Health, Welfare and Sports; Dutch Ministry of Health; Dutch Prevention Funds; LK Research Funds; Dutch ZON (Zorg Onderzoek Nederland); World Cancer Research Fund (WCRF); Statistics Netherlands (The Netherlands); Swedish Cancer Society; Swedish Scientific Council; Regional Government of Skane, Sweden; Nordforsk-Centre of Excellence programme. Some authors are partners of ECNIS, a network of excellence of the 6FP of the EC. RZ-R is thankful for a postdoctoral programme Fondo de Investigación Sanitaria (FIS; no. CD09/00133) from the Spanish Ministry of Science and Innovation. 


\section{CONFLICT OF INTEREST}

The authors declare no conflict of interest.

\section{REFERENCES}

Allen NE, Appleby PN, Key TJ, Bueno-de-Mesquita HB, Ros MM, Kiemeney LA, Tjønneland A, Roswall N, Overvad K, Weikert S, Boeing H, Chang-Claude J, Teucher B, Panico S, Sacerdote C, Tumino R, Palli D, Sieri S, Peeters P, Quirós JR, Jakszyn P, Molina-Montes E, Chirlaque MD, Ardanaz E, Dorronsoro M, Khaw KT, Wareham N, Ljungberg B, Hallmans G, Ehrnström R, Ericson U, Gram IT, Parr CL, Trichopoulou A, Karapetyan T, Dilis V, Clavel-Chapelon F, Boutron-Ruault MC, Fagherrazzi G, Romieu I, Gunter MJ, Riboli E (2013) Macronutrient intake and risk of urothelial cell carcinoma in the European prospective investigation into cancer and nutrition. Int J Cancer 132: 635-644.

Arts IC, Jacobs Jr. DR, Gross M, Harnack LJ, Folsom AR (2002) Dietary catechins and cancer incidence among postmenopausal women: the Iowa Women's Health Study (United States). Cancer Causes Control 13: 373-382.

Bosetti C, Rossi M, McLaughlin JK, Negri Talamini R, Lagiou P, Montella M, Ramazzotti V, Franceschi S, LaVecchia C (2007) Flavonoids and the risk of renal cell carcinoma. Cancer Epidemiol Biomarkers Prev 16: 98-101.

Buchner FL, Bueno-de-Mesquita HB, Ros MM, Kampman E, Egevad L, Overvad K, Raaschou-Nielsen O, Tjønneland A, Roswall N, Clavel-Chapelon F, Boutron-Ruault MC, Touillaud M, Chang-Claude J, Kaaks R, Boeing H, Weikert S, Trichopoulou A, Lagiou P, Trichopoulos D, Palli D, Sieri S, Vineis P, Tumino R, Panico S, Vrieling A, Peeters PH, van Gils CH, Lund E, Gram IT, Engeset D, Martinez C, Gonzalez CA, Larrañaga N, Ardanaz E, Navarro C, Rodríguez L, Manjer J, Ehrnström RA, Hallmans G, Ljungberg B, Allen NE, Roddam AW, Bingham S, Khaw KT, Slimani N, Boffetta P, Jenab M, Mouw T, Michaud DS, Kiemeney LA, Riboli E (2009) Consumption of vegetables and fruit and the risk of bladder cancer in the European Prospective Investigation into Cancer and Nutrition. Int J Cancer 125: 2643-2651.

Buckland G, Ros MM, Roswall N, Bueno-de-Mesquita HB, Travier N, Tjonneland A, Kiemeney LA, Sacerdote C, Tumino R, Ljungberg B, Gram IT, Weiderpass E, Skeie G, Malm J, Ehrnström R, Chang-Claude J, Mattiello A, Agnoli C, Peeters PH, Boutron-Ruault MC, Fagherazzi G, Clavel-Chapelon F, Nilsson LM, Amiano P, Trichopoulou A, Oikonomou E, Tsiotas K, Sánchez MJ, Overvad K, Quirós JR, Chirlaque MD, Barricarte A, Key TJ, Allen NE, Khaw KT, Wareham N, Riboli E, Kaaks R, Boeing H, Palli D, Romieu I, Romaguera D, Gonzalez CA (2014) Adherence to the Mediterranean diet and risk of bladder cancer in the EPIC cohort study. Int J Cancer 134: 2504-2511.

Burger M, Catto JW, Dalbagni G, Grossman HB, Herr H, Karakiewicz P, Kassouf W, Kiemeney LA, La Vecchia C, Shariat S, Lotan Y (2013) Epidemiology and risk factors of urothelial bladder cancer. Eur Urol 63: 234-241.

Chung FL, Morse MA, Eklind KI, Xu Y (1993) Inhibition of tobacco-specific nitrosamine-induced lung tumorigenesis by compounds derived from cruciferous vegetables and green tea. Ann N Y Acad Sci 686: 186-201.

Crozier A, Lean MEJ, McDonald MS, Black C (1997) Quantitative analysis of the flavonoid content of commercial tomatoes, onions, lettuces, and celery. J Agric Food Chem 45: 590-595.

Ferlay J, Shin HR, Bray F, Forman D, Mathers C, Parkin DM (2010) Estimates of worldwide burden of cancer in 2008: GLOBOCAN 2008. Int J Cancer 127: 2893-2917.

Garcia R, Gonzalez CA, Agudo A, Riboli E (1999) High intake of specific carotenoids and flavonoids does not reduce the risk of bladder cancer. Nutr Cancer 35: 212-214.

Giacosa A, Barale R, Bavaresco L, Gatenby P, Gerbi V, Janssens J, Johnston B, Kas K, La Vecchia C, Mainguet P, Morazzoni P, Negri E, Pelucchi C, Pezzotti M, Rondanelli M (2013) Cancer prevention in Europe: the Mediterranean diet as a protective choice. Eur J Cancer Prev 22: 90-95.

Guarrera S, Sacerdote C, Fiorini L, Marsala R, Polidoro S, Gamberini S, Saletta F, Malaveille C, Talaska G, Vineis P, Matullo G (2007) Expression of DNA repair and metabolic genes in response to a flavonoid-rich diet. Br J Nutr 98: 525-533.

Kiemeney LA, Thorlacius S, Sulem P, Geller F, Aben KK, Stacey SN, Gudmundsson J, Jakobsdottir M, Bergthorsson JT, Sigurdsson A, Blondal T, Witjes JA, Vermeulen SH, Hulsbergen-van de Kaa CA, Swinkels DW,
Ploeg M, Cornel EB, Vergunst H, Thorgeirsson TE, Gudbjartsson D, Gudjonsson SA, Thorleifsson G, Kristinsson KT, Mouy M, Snorradottir S, Placidi D, Campagna M, Arici C, Koppova K, Gurzau E, Rudnai P, Kellen E, Polidoro S, Guarrera S, Sacerdote C, Sanchez M, Saez B, Valdivia G, Ryk C, de Verdier P, Lindblom A, Golka K, Bishop DT, Knowles MA, Nikulasson S, Petursdottir V, Jonsson E, Geirsson G, Kristjansson B, Mayordomo JI, Steineck G, Porru S, Buntinx F, Zeegers MP, Fletcher T, Kumar R, Matullo G, Vineis P, Kiltie AE, Gulcher JR, Thorsteinsdottir U, Kong A, Rafnar T, Stefansson K (2008) Sequence variant on 8q24 confers susceptibility to urinary bladder cancer. Nat Genet 40: 1307-1312.

Malaveille C, Fiorini L, Bianchini M, Davico L, Bertinetti S, Allegro G, Hautefeuille A, Sacerdote C, Vineis P (2004) Randomized controlled trial of dietary intervention: association between level of urinary phenolics and anti-mutagenicity. Mutat Res 561: 83-90.

Manach C, Williamson G, Morand C, Scalbert A, Remesy C (2005) Bioavailability and bioefficacy of polyphenols in humans. I. Review of 97 bioavailability studies. Am J Clin Nutr 81(Suppl 1): 230S-242S.

Margetts BM, Pietinen P (1997) European Prospective Investigation into Cancer and Nutrition: validity studies on dietary assessment methods. Int J Epidemiol 26(Suppl 1): S1-S5.

Neveu V, Perez-Jimenez J, Vos F, Crespy V, du Chaffaut L, Mennen L, Knox C, Eisner R, Cruz J, Wishart D, Scalbert A (2010) Phenol-Explorer: an online comprehensive database on polyphenol contents in foods. Database (Oxford) 2010: bap024.

Park SY, Ollberding NJ, Woolcott CG, Wilkens LR, Henderson BE, Kolonel LN (2013) Fruit and vegetable intakes are associated with lower risk of bladder cancer among women in the Multiethnic Cohort Study. J Nutr 143: 1283-1292.

Pelucchi C, Bosetti C, Rossi M, Negri E, La Vecchia C (2009) Selected aspects of Mediterranean diet and cancer risk. Nutr Cancer 61: 756-766.

Perez-Jimenez J, Neveu V, Vos F, Scalbert A (2010) Identification of the 100 richest dietary sources of polyphenols: an application of the Phenol-Explorer database. Eur J Clin Nutr 64(Suppl 3): S112-S120.

Riboli E, Kaaks R (1997) The EPIC Project: rationale and study design. European Prospective Investigation into Cancer and Nutrition. Int J Epidemiol 26(Suppl 1): S6-14.

Riboli E, Hunt KJ, Slimani N, Ferrari P, Norat T, Fahey M, Charrondière UR, Hémon B, Casagrande C, Vignat J, Overvad K, Tjønneland A, Clavel-Chapelon F, Thiébaut A, Wahrendorf J, Boeing H, Trichopoulos D, Trichopoulou A, Vineis P, Palli D, Bueno-De-Mesquita HB, Peeters PH, Lund E, Engeset D, González CA, Barricarte A, Berglund G, Hallmans G, Day NE, Key TJ, Kaaks R, Saracci R (2002) European Prospective Investigation into Cancer and Nutrition (EPIC): study populations and data collection. Public Health Nutr 5: 1113-1124.

Rockenbach L, Bavaresco L, Fernandes Farias P, Cappellari AR, Barrios CH, Bueno Morrone F, Oliveira Battastini AM (2013) Alterations in the extracellular catabolism of nucleotides are involved in the antiproliferative effect of quercetin in human bladder cancer T24 cells. Urol Oncol 31: 1204-1211.

Ros MM, Bueno-de-Mesquita HB, Kampman E, Aben KK, Büchner FL, Jansen EH, van Gils CH, Egevad L, Overvad K, Tjønneland A, Roswall N, Boutron-Ruault MC, Kvaskoff M, Perquier F, Kaaks R, Chang-Claude J, Weikert S, Boeing H, Trichopoulou A, Lagiou P, Dilis V, Palli D, Pala V, Sacerdote C, Tumino R, Panico S, Peeters PH, Gram IT, Skeie G, Huerta JM, Barricarte A, Quirós JR, Sánchez MJ, Buckland G, Larrañaga N, Ehrnström R, Wallström P, Ljungberg B, Hallmans G, Key TJ, Allen NE, Khaw KT, Wareham N, Brennan P, Riboli E, Kiemeney LA (2010) Plasma carotenoids and vitamin $\mathrm{C}$ concentrations and risk of urothelial cell carcinoma in the European Prospective Investigation into Cancer and Nutrition. Am J Clin Nutr 96: 902-910.

Ros MM, Bueno-de-Mesquita HB, Kampman E, Büchner FL, Aben KK, Egevad L, Overvad K, Tjønneland A, Roswall N, Clavel-Chapelon F, Boutron-Ruault MC, Morois S, Kaaks R, Teucher B, Weikert S, von Ruesten A, Trichopoulou A, Naska A, Benetou V, Saieva C, Pala V, Ricceri F, Tumino R, Mattiello A, Peeters PH, van Gils CH, Gram IT, Engeset D, Chirlaque MD, Ardanazx E, Rodríguez L, Amanio P, Gonzalez CA, Sánchez MJ, Ulmert D, Ernström R, Ljungberg B, Allen NE, Key TJ, Khaw KT, Wareham N, Slimani N, Romieu I, Kiemeney LA, Riboli E (2012) Fruit and vegetable consumption and risk of aggressive and non-aggressive urothelial cell carcinomas in the European Prospective Investigation into Cancer and Nutrition. Eur J Cancer 48: 3267-3277.

Russo M, Spagnuolo C, Tedesco I, Bilotto S, Russo GL (2012) The flavonoid quercetin in disease prevention and therapy: facts and fancies. Biochem Pharmacol 83: 6-15. 
Sacerdote C, Matullo G, Polidoro S, Gamberini S, Piazza A, Karagas MR, Rolle L, De Stefanis P, Casetta G, Morabito F, Vineis P, Guarrera S (2007) Intake of fruits and vegetables and polymorphisms in DNA repair genes in bladder cancer. Mutagenesis 22: 281-285.

Skeie G, Braaten T, Hjartaker A, Lentjes M, Amiano P, Jakszyn P, Pala V, Palanca A, Niekerk EM, Verhagen H, Avloniti K, Psaltopoulou T, Niravong M, Touvier M, Nimptsch K, Haubrock J, Walker L, Spencer EA, Roswall N, Olsen A, Wallström P, Nilsson S, Casagrande C, Deharveng G, Hellström V, Boutron-Ruault MC, Tjønneland A, Joensen AM, Clavel-Chapelon F, Trichopoulou A, Martinez C, Rodríguez L, Frasca G, Sacerdote C, Peeters PH, Linseisen J, Schienkiewitz A, Welch AA, Manjer J, Ferrari P, Riboli E, Bingham S, Engeset D, Lund E, Slimani N (2009) Use of dietary supplements in the European Prospective Investigation into Cancer and Nutrition calibration study. Eur J Clin Nutr 63(Suppl 4): S226-S238.

Slimani N, Deharveng G, Unwin I, Southgate DA, Vignat J, Skeie G, Salvini S, Parpinel M, Møller A, Ireland J, Becker W, Farran A, Westenbrink S, Vasilopoulou E, Unwin J, Borgejordet A, Rohrmann S, Church S, Gnagnarella P, Casagrande C, van Bakel M, Niravong M, Boutron-Ruault MC, Stripp C, Tjønneland A, Trichopoulou A, Georga K, Nilsson S, Mattisson I, Ray J, Boeing H, Ocké M, Peeters PH, Jakszyn P, Amiano P, Engeset D, Lund E, de Magistris MS, Sacerdote C, Welch A, Bingham S, Subar AF, Riboli E (2007) The EPIC nutrient database project (ENDB): a first attempt to standardize nutrient databases across the 10 European countries participating in the EPIC study. Eur J Clin Nutr 61: 1037-1056.

Steele VE, Kelloff GJ, Balentine D, Boone CW, Mehta R, Bagheri D, Sigman CC, Zhu S, Sharma S (2000) Comparative chemopreventive mechanisms of green tea, black tea and selected polyphenol extracts measured by in vitro bioassays. Carcinogenesis 21: 63-67.

Sun F, Zheng XY, Ye J, Wu TT, Wang JI, Chen W (2012) Potential anticancer activity of myricetin in human T24 bladder cancer cells both in vitro and in vivo. Nutr Cancer 64: 599-606.

Talaska G, Al-Zoughool M, Malaveille C, Fiorini L, Schumann B, Vietas J, Peluso M, Munnia A, Bianchini M, Allegro G, Matullo G, Sacerdote C, Vineis P (2006) Randomized controlled trial: effects of diet on DNA damage in heavy smokers. Mutagenesis 21: 179-183.

U.S.Departament of Agriculture (2004) USDA Database for the Proanthocyanidin Content of Selected Foods. USDA: Beltsville, MD.

U.S.Departament of Agriculture (2007) USDA Database for the Flavonoid Content of Selected Foods. USDA: Beltsville. MD.

U.S.Departament of Agriculture (2008) USDA Database for the Isoflavone Content of Selected Foods. USDA: Beltsville, MD.

Wang X, Lin YW, Wang S, Wu J, Mao QQ, Zheng XY, Xie LP (2013) A meta-analysis of tea consumption and the risk of bladder cancer. Urol Int 90: $10-16$

Ward HA, Kuhnle GG, Mulligan AA, Lentjes MA, Luben RN, Khaw KT (2010) Breast, colorectal, and prostate cancer risk in the European Prospective Investigation into Cancer and Nutrition-Norfolk in relation to phytoestrogen intake derived from an improved database. Am J Clin Nutr 91: $440-448$.

Wareham NJ, Jakes RW, Rennie KL, Schuit J, Mitchell J, Hennings S, Day NE (2003) Validity and repeatability of a simple index derived from the short physical activity questionnaire used in the European Prospective Investigation into Cancer and Nutrition (EPIC) study. Public Health Nutr 6: $407-413$.

Wilson RT, Wang J, Chinchilli V, Richie JP, Virtamo Jarmo, Moore LE, Albanes D (2009) Fish, vitamin D, and flavonoids in relation to renal cell cancer among smokers. Am J Epidemiol 170: 717-729.

World Research Cancer Fund, American Institute for Cancer Research (2007) Food, Nutrition, Physical Activity and the Prevention of Cancer: A Global Perspective Washington, DC.

Xiao ZP, Peng ZY, Peng MJ, Yan WB, Ouyang YZ, Zhu HL (2011) Flavonoids health benefits and their molecular mechanism. Mini Rev Med Chem 11: 169-177.

Xie F, Su M, Qiu W, Zhang M, Guo Z, Su B, Liu J, Li X, Zhou L (2013) Kaempferol promotes apoptosis in human bladder cancer cells by inducing the tumor suppressor, PTEN. Int J Mol Sci 14: 21215-21226.

Yao LH, Jiang YM, Shi J, Tomás-Barberán FA, Datta N, Singanusong R, Chen SS (2004) Flavonoids in food and their health benefits. Plant Foods Hum Nutr 59: 113-122.

Zamora-Ros R, Knaze V, Lujan-Barroso L, Kuhnle GG, Mulligan AA, Touillaud M, Slimani N, Romieu I, Powell N, Tumino R, Peeters PH, de Magistris MS, Ricceri F, Sonestedt E, Drake I, Hjartåker A, Skie G, Mouw T, Wark PA, Romaguera D, Bueno-de Mesquita HB, Ros M, Molina E, Sieri S, Quirós JR, Huerta JM, Tjønneland A, Halkjær J, Masala G, Teucher B, Kaas R, Travis RC, Dilis V, Benetou V, Trichopoulou A, Amiano P, Ardanaz E, Boeing H, Förster J, Clavel-Chapelon F, Fagherazzi G, Perquier F, Johansson G, Johansson I, Cassidy A, Overvad K, González CA (2012) Dietary intakes and food sources of phytoestrogens in the European Prospective Investigation into Cancer and Nutrition (EPIC) 24-hour dietary recall cohort. Eur J Clin Nutr 66: 932-941.

Zamora-Ros R, Knaze V, Lujan-Barroso L, Romieu I, Scalbert A, Slimani N, Hjartåker A, Engeset D, Skeie G, Overvad K, Bredsdorff L, Tjønneland A, Halkjær J, Key TJ, Khaw KT, Mulligan AA, Winkvist A, Johansson I, Bueno-de-Mesquita HB, Peeters PH, Wallström P, Ericson U, Pala V, de Magistris MS, Polidoro S, Tumino R, Trichopoulou A, Dilis V, Katsoulis M, Huerta JM, Martínez V, Sánchez MJ, Ardanaz E, Amiano P, Teucher B, Grote V, Bendinelli B, Boeing H, Förster J, Touillaud M, Perquier F, Fagherazzi G, Gallo V, Riboli E, González CA (2013) Differences in dietary intakes, food sources, and determinants of total flavonoids between Mediterranean and non-Mediterranean countries participating in the European Prospective Investigation into Cancer and Nutrition (EPIC) study. Br J Nutr 109: 1498-1507.

Zamora-Ros R, Touillaud M, Rothwell JA, Romieu I, Scalbert A (2014) Measuring exposure to the polyphenol metabolome in observational epidemiologic studies: current tools and applications and their limits. Am J Clin Nutr 100: 11-26.

This work is published under the standard license to publish agreement. After 12 months the work will become freely available and the license terms will switch to a Creative Commons AttributionNonCommercial-Share Alike 3.0 Unported License.

Supplementary Information accompanies this paper on British Journal of Cancer website (http://www.nature.com/bjc) 